\title{
Study of Interference Effect of Wind Pressure on Roof of Multi-storey Residential Buildings
}

\author{
Xiao Luan, Peng Huang* \\ State Key Laboratory of Disaster Reduction in Civil Engineering, Tongii University, Shanghai, China \\ Email address: \\ 18220530350@163.com (Xiao Luan), huangtju@tongji.edu.cn (Peng Huang) \\ ${ }^{*}$ Corresponding author
}

To cite this article:

Xiao Luan, Peng Huang. Study of Interference Effect of Wind Pressure on Roof of Multi-storey Residential Buildings. Science Discovery. Vol. 9, No. 1, 2021, pp. 13-20. doi: 10.11648/j.sd.20210901.13

Received: January 5, 2021; Accepted: January 29, 2021; Published: January 30, 2021

\begin{abstract}
In this paper, a series of wind tunnel experiments were carried out on the rigid model of 5-6 storey residential buildings to study the influence of the location, spacing and wind direction of the buildings on the roof wind pressure of multi-storey residential buildings. The test results show that the maximum average wind pressure and extreme negative wind pressure of a single building occur in the roof ridge area at the corner and both ends of the roof, taking a multi-storey building with length $\times$ width $\times$ height of $60 \mathrm{~m} \times 12 \mathrm{~m} \times 20 \mathrm{~m}$ as an example. For the buildings with three rows and three columns, when the building is located at the corner, the most unfavorable wind direction is $30^{\circ}, 210^{\circ}$, and $330^{\circ}$. The most unfavorable extreme value of negative wind pressure is -8.72 . The negative wind pressure coefficient of the most unfavorable extreme value of the building roof at the corner position and the middle position of the first row decreased with the length-width ratio and the spacing increased. The negative wind pressure coefficient of the most unfavorable extreme value of the building roof at the edge and middle of the second row did not change significantly with the increase of length-width ratio but increased with the increase of space. In the corner area of the roof, the interference factor of the corner position and the middle position of the first row decreased with the space, while the interference factor of the edge position and the middle position of the second row increased with the space. The variation trend of the middle area of the roof is the same as that of the corner area, but the interference factor is generally small. The interference factor of the wall area is always greater than 1.0.
\end{abstract}

Keywords: Group Building, Typical Community, Extreme Negative Wind Pressure, Interference Factor

\section{多层小区建筑群屋面风压干扰效应研究}

\author{
奕晓, 黄鹏* \\ 同济大学土木工程防灾国家重点实验室, 上海, 中国 \\ 邮箱 \\ 18220530350@163.com (栾晓), huangtju@tongji.edu.cn (黄鹏)
}

\begin{abstract}
摘要: 本文通过对5-6层居民建筑的刚性模型进行风洞实验, 研究了建筑物的位置、前后间距和风向角对多层小区建筑 群屋面风压的干扰影响规律。试验结果表明：以长 $\times$ 宽 $\times$ 高为 $60 \mathrm{~m} \times 12 \mathrm{~m} \times 20 \mathrm{~m}$ 的多层小区建筑为例, 单体建筑的平均风 压和极值负风压的最大值均出现在屋盖的角部和两端部的屋脊区域。对于三排三列的建筑群, 当建筑位于角部位置时 最不利, $30^{\circ}, 210^{\circ}, 330^{\circ}$ 为三个最不利风向角, 最不利极值负风压为 -8.72 ; 角部位置、第一排中间位置建筑屋面的最 不利极值负风压系数随长宽比减小而减小，随间距增大而减小; 第二排边缘位置和中部位置建筑屋面的最不利极值负 风压系数随长宽比增大无明显变化, 随间距增大而增大。屋面角部区域, 角部位置、第一排中间位置建筑的干扰因子 随间距的增大而减小, 第二排边缘位置和中部位置的干扰因子随间距增大而增大; 屋面中部区域变化趋势同角部区域, 但干扰因子整体偏小; 墙面区域的干扰因子始终大于 1.0 。
\end{abstract}


关键词: 群体建筑, 典型小区, 极值负风压, 干扰因子

\section{1. 引言}

多层小区通常以群体形式分布, 相邻建筑物之间的气流 干扰效应明显, 受扰建筑的风压分布明显区别于单体建筑, 忽略这部分干扰效应将会出现围护结构的部分位置存在安 全隐患, 部分位置的设计又过于保守, 因此研究多层小区的 风荷载干扰效应意义重大。目前国内外学者对高层的干扰效 应和低矮建筑的群体干扰效应进行了大量研究, 但针对 5-6 层, 高度约为 20 米, 长宽比较大的多层住宅小区建筑群的干 扰尚待深入研究。Tsutsumi, T. Katayama[8]针对常规的建筑 群的交错排列和正交排列两种布局进行实验，同时考虑了风 向影响。结果表明, 群体干扰下的建筑物风压系数要比单体 建筑的风压系数要高。E.C.English [10]基于现有的风洞试验 的数据, 使用人工神经网络的方法来研究两栋建筑之间的干 扰和遮挡作用，并且用新定义的“干扰因子”来量化两栋建筑 之间的干扰和遮挡作用。Chang C H, Meroney R N[13]通过研 究间距对低矮房屋表面风压的影响, 得出遮蔽效应房屋间距 与房屋高度的比值和上游干扰建筑数量。Yong Chul Kim $[9$, 15]通过研究大量周边建筑对典型低矮建筑风压的影响得出, 尽管平均风压系数随着周边建筑面积密度的增加而减小, 但 当测点位于来流下游时, 其最大值和最小系数均大于单体建 筑, 并引入分区干扰因子, 研究了目标低层建筑风荷载的估 算方法。Yong C K, Tamura Y [11]研究了建筑面积密度和建 筑物间距的影响, 发现特别是屋盖边缘的风压不会随着建筑 物面积密度的增大而减小。Sun Y, Tamura Y, Quan Y[12] 进行风洞试验研究了周边建筑对中心矩形建筑的干扰, 结果 表明边建筑对中心矩形建筑的干扰非常显著, 与单体建筑相 比, 群体建筑的干扰会使风压系数峰值增大一倍。Kim [14] 通过研究大量相同周边建筑对目标建筑的干扰效应和遮蔽 效应, 发现最大风压系数绝对值比单体低矮建筑大很多, 目 标建筑越靠近下游, 其风压越小。黄鹏[4]总结了不同国家规 范中对干扰效应的研究成果。全涌[3]通过研究周边建筑对低 矮就按住屋面风荷载的干扰因子发现, 最大局部负风压干扰 因子随周边建筑密度增大而减小, 随周边建筑相对高度增大 而减小。骆盼育[5]通过研究大跨平屋盖群体干扰效应, 发现 小间距时呈现遮挡效应, 间距增大时个别位置会出现局部放 大效应。乔常贵[1]研究了中高层住宅区建筑群在不同的间距 下的风荷载特性。研究发现, 当间距 $\mathrm{Sx}$ 小于 $4 \mathrm{~b}$ 时, 干扰总体 呈现为遮挡效应, 建筑相邻侧面由于“狭管效应”, 风速有着 不同程度加强。成俊凯[2]研究了纵横排列的高层住宅小区的 干扰效应, 发现角部建筑遮挡效应小于中部建筑, 但角部建 筑的极值负风压呈现绝对值增大现象。程行[6]通过风洞试 验研究了不同坡度与长宽比的双坡屋面低矮房屋建筑群的 风荷载干扰效应。结果表明, 在 $45^{\circ}$ 风向下坡度为 $35^{\circ}$ 建筑物 屋面背风区域最不利风压系数增幅达 $200 \%$ 。

目前国内有大量的多层小区，由于此类小区多为有 30 年以上历史的老旧小区, 屋面和门窗等围护结构老化, 在 强风作用下很容易发生破坏, 对居民的生活产生不利影
响。本文通过对5-6层居民建筑的刚性模型风洞实验， 研究了建筑物屋面和墙面风压分布规律以及建筑物位置、 前后间距和风向角对多层小区建筑群屋面风压的干扰影 响规律, 给出了典型位置的最不利风向角和各个工况的最 不利干扰因子。

\section{2. 实验概况}

\section{1. 风洞概况}

本次课题采用刚性模型同步测压试验对建筑之间的 干扰效应进行系统研究。刚性模型测压试验是在同济大学 风洞实验室TJ-2号大气边界层建筑风洞内完成的。TJ-2号 边界层风洞是横向回流式低速风洞, 其截面形式为矩形, 风洞的试验段尺寸为 $3 \mathrm{~m}$ 宽、 $2.5 \mathrm{~m}$ 高、 $15 \mathrm{~m}$ 长。试验风速范 围从 $0.5 \mathrm{~m} / \mathrm{s} \sim 68 \mathrm{~m} / \mathrm{s}$, 在试验段的流畅风速的不均匀性 $<1 \%$, 湍流度 $<0.46 \%$, 平均气流偏角 $<0.5^{\circ}$, 整个流场性能良好。

\section{2. 风场模拟}

本次实验模拟的风场为中国建筑结构荷载规范[7]中的 $\mathrm{B}$ 类地貌。本次实验在风场上游设置两个尖䢃, 一根横栅 以及粗粘元来模拟风场, 风场模拟结果如图1。

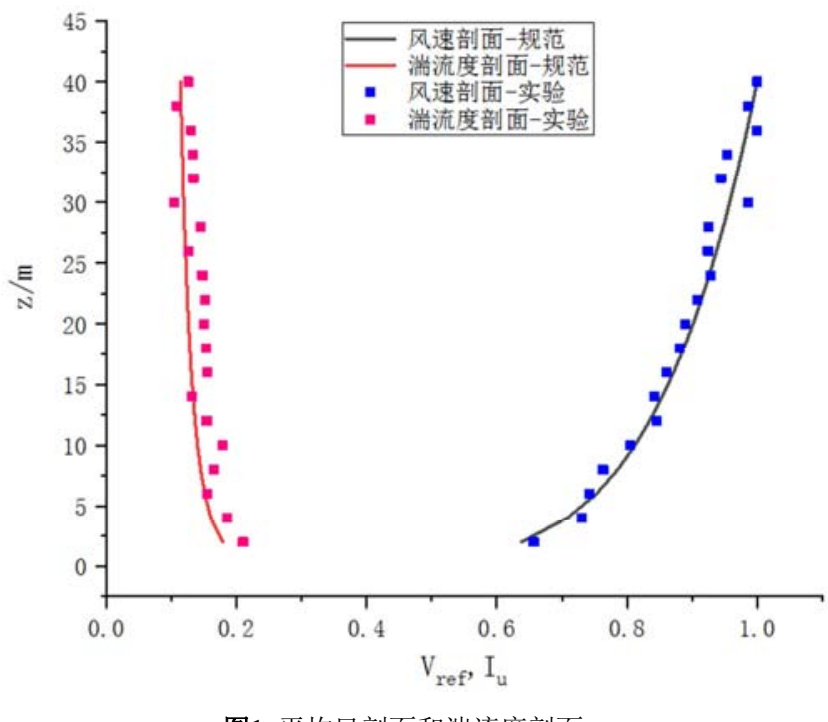

图1 平均风剖面和湍流度剖面。

\section{3. 实验模型及工况}

根据我国此类高层小区的实际情况, 对南北方的典型 城市的高层小区建筑单体的实际尺寸及排布方式进行大 量的考察并进行统计分析, 建筑群体选择三排三列行列式 布局, 坡度选择典型的 $9.4^{\circ}$ 和 $18.4^{\circ}$ 两种, 六层小区建筑的 屋檐高度取为 $20 \mathrm{~m}$, 长 $\times$ 宽 $\times$ 高分别定为 $36 \mathrm{~m} \times 12 \mathrm{~m} \times 20 \mathrm{~m}$, $48 \mathrm{~m} \times 12 \mathrm{~m} \times 20 \mathrm{~m}, 60 \mathrm{~m} \times 12 \mathrm{~m} \times 20 \mathrm{~m}$, 根据南北方纬度的不同， 考虑冬季太阳高度角的差异, 为保证南北不同地区对光照 
的要求, 建筑的前后间距分别取 $20 \mathrm{~m} 、 35 \mathrm{~m}$ 和 $50 \mathrm{~m}$, 左右 间距根据行车要求等实际情况取 $10 \mathrm{~m}$ 。

本次实验的缩尺比选为 $1: 100$, 满足风洞试验最小阻 塞比的要求。模型对应的高度为 $20 \mathrm{~cm}$, 长 $\times$ 宽 $\times$ 高为 $36 \mathrm{~cm} \times 12 \mathrm{~cm} \times 20 \mathrm{~cm}, 48 \mathrm{~cm} \times 12 \mathrm{~cm} \times 20 \mathrm{~cm}, 60 \mathrm{~cm} \times 12 \mathrm{~cm} \times 20 \mathrm{~cm}$, 模型的前后间距分别为 $20 \mathrm{~cm}, 35 \mathrm{~cm}, 50 \mathrm{~cm}$, 左右间距为 $10 \mathrm{~cm}$, 模型示意图如图2所示。

为了方便研究, 图3给出了本次实验屋面及墙面的编 号。具体模型尺寸见表 1 。

模型的墙面和坡屋面均匀布满测点, 用来测量试验条 件下模型表面的风压系数, 测点布置遵循对称均匀, 边缘 和角部加密原则。对于 $\mathrm{L}=36 \mathrm{~cm}, 48 \mathrm{~cm}, 60 \mathrm{~cm}$ 模型表面分 别共布置 $174,202,230$ 个测点, 每个模型的测点布置方 式均一致, 且所有测点均为单面测点。以长宽比 $60 \times 12$, 坡度为 $18.2^{\circ}$ 的模型为例, 表面测点及编号布置如图4所示 （屋盖坡度为 $9.4^{\circ}$ 和 $18.4^{\circ}$ 的测点布置和编号均一致）。模 型试验图见图5。

本次实验共有 3 (3种长宽比) $\times 2$ (两种坡度) $=6$ 个大 工况, 除单体情况, 每种大工况还分别研究三种间距

$(20 \mathrm{~cm}, 35 \mathrm{~cm}, 50 \mathrm{~cm})$ 以及四个位置的风压干扰效应。建筑 位置示意图见图6, 角部位置本文定义为位置1, 第一排中 部位置定义为位置2, 第二排边缘位置定义为位置3, 中部 位置定义为位置4。

为了更好的研究屋面及墙面干扰因子, 本文将建筑物 屋面分成角部区域 4 个分区, 屋面中间区域 2 个分区, 墙面 分成 2 个分区, 六种模型均采用统一分区原则。以模型 1 为例, 分区编号详见图7。

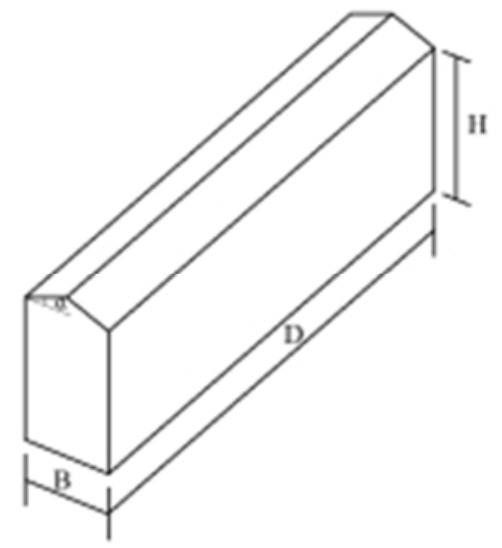

图2 模型示意图。

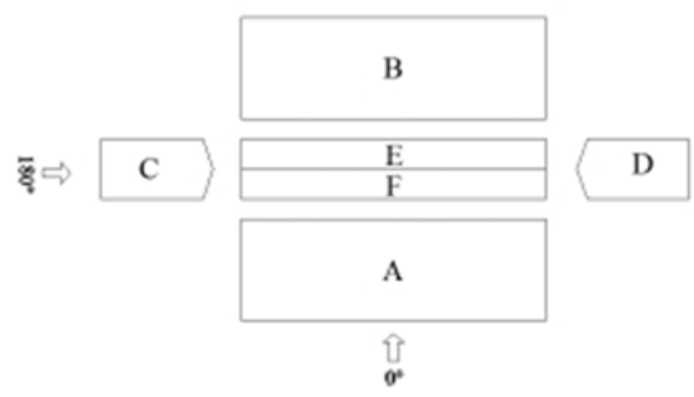

图3 墙面及屋面编号。
表1 模型尺寸。

\begin{tabular}{|c|c|c|c|c|}
\hline $\begin{array}{l}\text { 模 型 } \\
\text { 编号 }\end{array}$ & 坡度 $\left(^{(}\right)$ & $\begin{array}{l}\text { 长 } \times \text { 宽 } \times \text { 高（屋檐高 } \\
\text { 度）(cm) }\end{array}$ & $\begin{array}{l}\text { 屋脊高度 } \\
(\mathbf{c m})\end{array}$ & 长宽比 \\
\hline 1 & $18.4(4: 12)$ & $60 \times 12 \times 20$ & 22 & $5: 1$ \\
\hline 2 & $18.4(4: 12)$ & $48 \times 12 \times 20$ & 22 & 4: 1 \\
\hline 3 & $18.4(4: 12)$ & $36 \times 12 \times 20$ & 22 & 3: 1 \\
\hline 4 & $9.4(2: 12)$ & $60 \times 12 \times 20$ & 21 & $5: 1$ \\
\hline 5 & $9.4(2: 12)$ & $48 \times 12 \times 20$ & 21 & 4: 1 \\
\hline 6 & $9.4(2: 12)$ & $36 \times 12 \times 20$ & 21 & 3: 1 \\
\hline
\end{tabular}

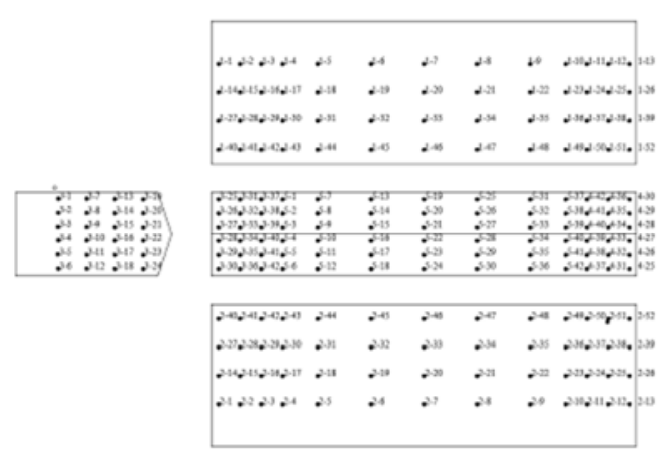

图4 测点布置示意图。

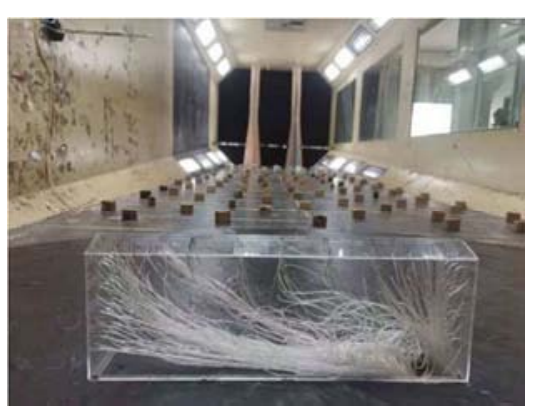

图5 模型试验图。

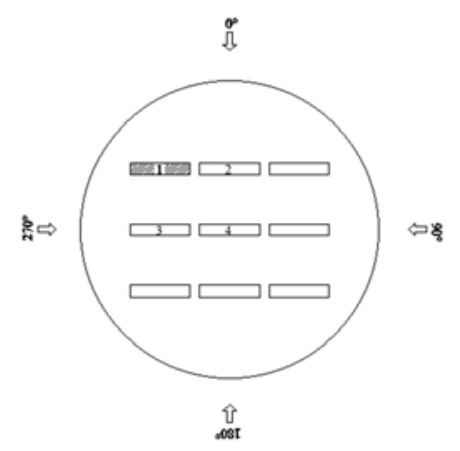

图6 建筑位置及风向角示意图。

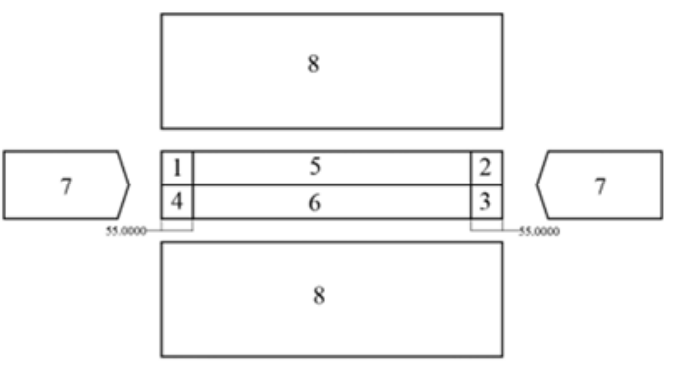

图7 屋面及墙面分区。 


\section{4. 数据处理}

(1) 瞬时风压系数

风压系数是风在建筑表面产生的实际压力与来流风 压的比值, 建筑模型表面的压力可用无量纲的瞬时压力系 数 $C_{P i}(t)$ (以梯度风压为参考风压的系数）, 表示为:

$$
C_{P i}(t)=\frac{P_{\mathrm{i}}(t)-P_{\infty}}{P_{0}-P_{\infty}}
$$

式中: $P_{\mathrm{i}}(t)$ - $\mathrm{t}$ 时刻作用在测点 $\mathrm{i}$ 处的风压; $P_{0}-$ 参考高度处的总压; $P_{\infty}$ 一参考高度处的静压。进行数据 处理得到平均风压系数 $\overline{C_{P i}}$ 和脉动风压系数 $\sigma_{P i}$ 。

极值风压系数

本文的极值风压计算方法采用Davenport的峰值因子 法, 表达式如下:

$$
\begin{aligned}
C_{P i \text { min }} & =\overline{C_{P i}}-g \sigma_{P \mathrm{i}} \\
C_{P i \text { max }} & =\overline{C_{P i}}+g \sigma_{P \mathrm{i}}
\end{aligned}
$$

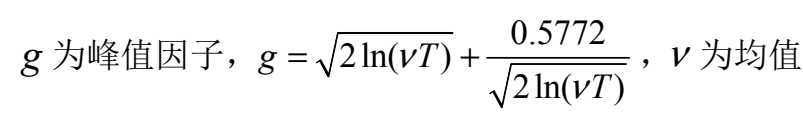
穿越率, 是随机数据穿越平均值的速率, $T$ 是观察时间。 极值风压干扰因子

$$
\begin{gathered}
I F_{\min }=\frac{C_{P i \text { min,interference }}}{C_{P i \text { min, issolation }}} \\
I F_{\max }=\frac{C_{P i \text { max,interference }}}{C_{P i \text { max,isolation }}}
\end{gathered}
$$

$C_{P i \text { max,isolation }} 、 C_{P i \text { min,isolation }}$ 为孤立建筑上分区 $\mathrm{i}$ 的所有 测点全风向角下的极大值和极小值风压系数, $C_{P i \text { max,interference }} 、 C_{P i \text { inin,interence }}$ 为有干扰时, 目标建筑上分 区 $\mathrm{i}$ 的所有测点全风向角下的极大值和极小值风压系数。

\section{3. 结果分析}

双坡屋面建筑，当坡度较小时往往是负压对屋面的破 坏起控制性作用。由于本实验的屋面坡度为 $9.4^{\circ}$ 和 $18.4^{\circ}$, 均为小坡度屋面, 因此本文重点研究建筑物屋面在干扰下 的负风压变化规律, 主要针对 $18.4^{\circ}$ 这一典型坡度的模型进 行研究。

\section{1. 单体风压特性分析}

图8给出模型 1 的单体建筑在全风向角下的平均风压 等值线图。平均风压系数最大值出现在屋盖 $\mathrm{E}$ 的角部, 大 小为 -3.34 , 风向角为 $210^{\circ}$; 墙面的平均风压系数最大值出 现在山墙 $\mathrm{D}$ 的角部, 大小为 -1.96 , 风向角为 $190^{\circ}$ 。从图 8 可以看出, 屋盖平均风压在四个角部和两个端部的屋脊区 域最为不利; 墙面平均负风压最大值出现在两个山墙同屋
盖相接的角部。屋面和墙面均呈现出较好的对称性, 表明 实验重复性好。

单体建筑全风向角下的极值负风压系数等值线图如 图9所示, 可以看出, 屋盖整体受到的吸力明显要比墙面 受到的吸力要大, 尤其是屋盖的四个角部, 此时屋盖角部 的极值负风压系数最大达到了 -8.19 , 风向角为 $210^{\circ}$; 两端 的屋脊区域也达到-6.00左右, 同样需要重点关注; 山墙的 极值负风压系数最大达到了 -5.20 , 出现的风向角为 $150^{\circ}$ 。

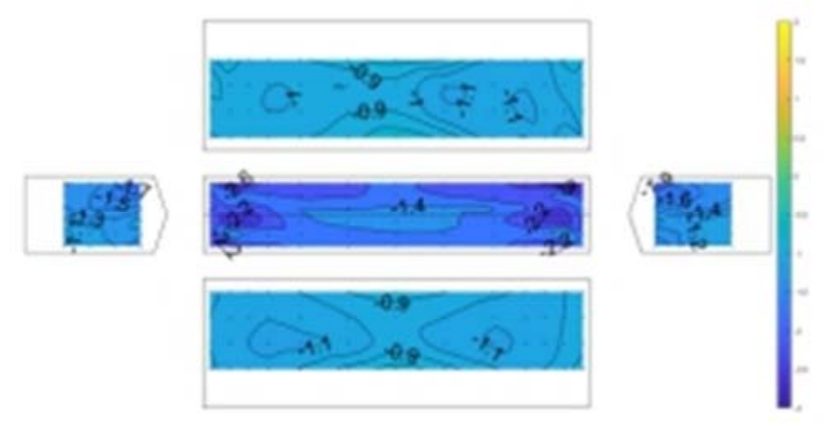

图8 全风向角下平均风压系数等值线图。

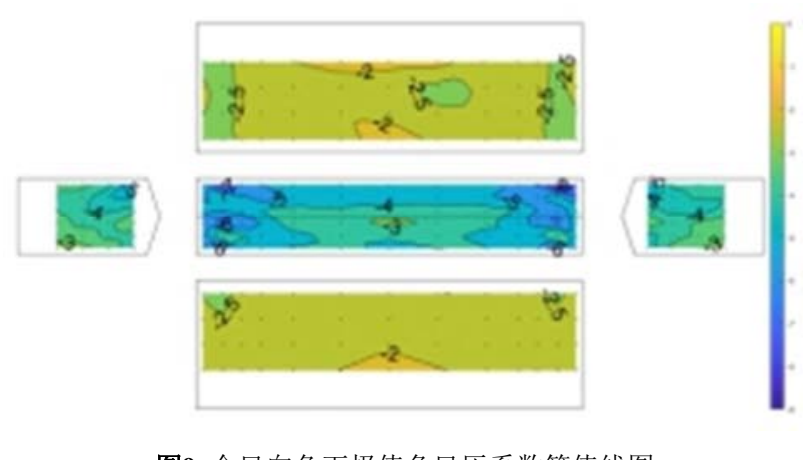

图9 全风向角下极值负风压系数等值线图。

\section{2. 最不利位置风压特性分析}

利用公式 (3) 求出屋面和墙面的极值负风压系数系 数后, 分析发现建筑物位于位置 1 , 间距 $20 \mathrm{~m}$ 时, 屋面和 墙面受到的极值负风压最大, 因此选取该工况 $0^{\circ}, 30^{\circ}$, $210^{\circ}$ 和 $330^{\circ}$ 四个最不利风向角进行单独分析, 如图 10 所示。 $0^{\circ}$ 风向角时, 整个屋盖受负压控制, 屋面 $\mathrm{F}$ 前缘极值 负压系数最大为 -5.74 , 出现在靠近山墙 $\mathrm{C}$ 的角部, 山墙 $\mathrm{C}$ 受到的负压要比山墙 $\mathrm{D}$ 偏大, 原因是由于周边建筑的存在, 山墙 $\mathrm{C}$ 和位置 2 处干扰建筑的山墙间隔较小, 形成狭道, 使 山墙 $\mathrm{C}$ 负压整体偏大。 $30^{\circ}$ 风向角时, 来流在屋面 $\mathrm{F}$ 左下角 部区域发生分离, 产生的漩涡直接作用在屋面的角部区域, 同时由于周边建筑的遮挡时的部分气流无法顺利通过, 导 致角部极值负压系数最大为 -8.72 , 大于单体建筑的最不利 极值负风压 (-8.19)。

$210^{\circ}$ 时, 气流在屋盖 $\mathrm{E}$ 的右上角部分离, 产生的漩浴 作用在屋盖 $\mathrm{F}$ 的端部, 使得屋盖 $\mathrm{E}$ 的角部和屋盖 $\mathrm{F}$ 的端部均 产生了较大负压, 最大负压达到了-6.52; 当风向角为 $330^{\circ}$ 时, 此时周边建筑均位于目标建筑下游, 来流在屋盖的角 部分离, 产生负压最大为-6.27, 屋盖风压分布同 $210^{\circ}$ 时有 较好的对称性, 但和 $30^{\circ}$ 风向角对比分析, 由于 $330^{\circ}$ 时山墙 
$\mathrm{D}$ 同侧缺少建筑干扰, 屋盖极值负风压系数最大值比 $30^{\circ}$ 的要小。

经过分析可以得出, 三行三列排布时, 位于位置一(角 部）的建筑物在斜风向 $\left(30^{\circ}, 210^{\circ}, 330^{\circ}\right)$ 时的屋盖角部
负风压比其他角度要更大, 特别是在 $30^{\circ}$ 风向角时, 气流 分离引起的浴旋脱落加上建筑干扰引起的浴旋, 两部分共 同作用会使迎风的角部负压最大, 此时为最不利情况, 在 进行围护结构的设计时可以考虑重点加强该部位。

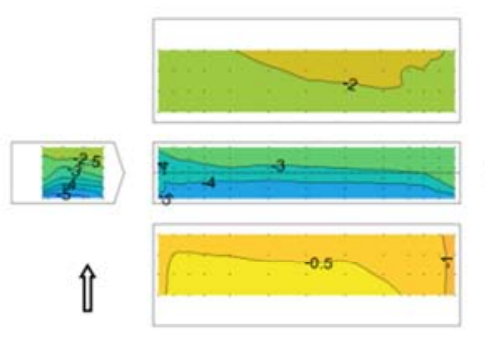

(a) 0 风向角

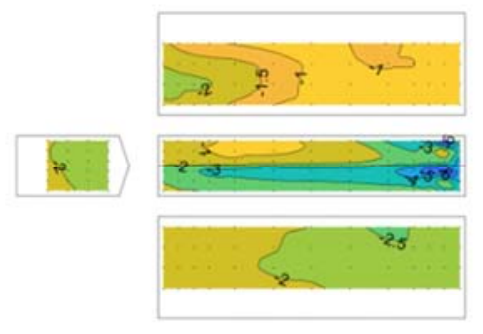

（c） $210^{\circ}$ 风向角
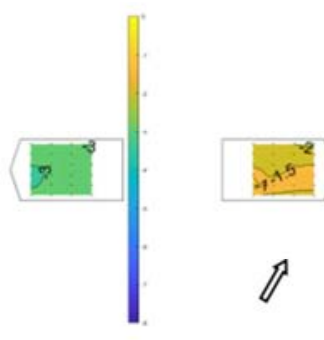

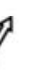

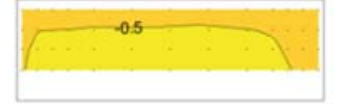

(b) $30^{\circ}$ 风向角

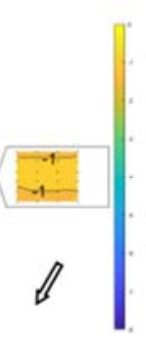

$22^{22}$

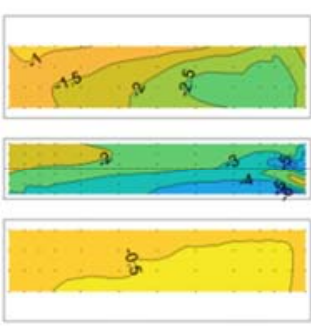

（d） $330^{\circ}$ 风向角

图10 位置1极值负风压系数等值线图。

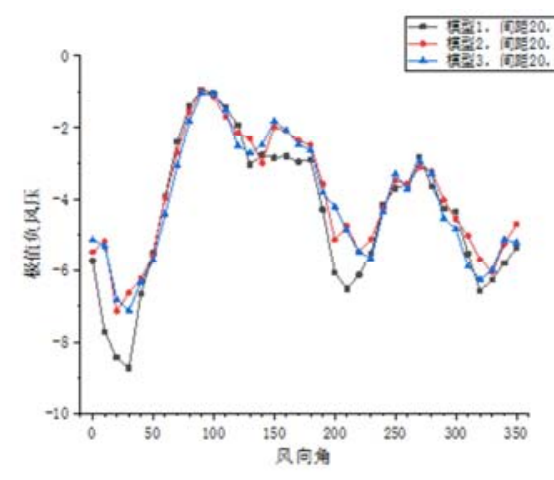

(a) 位置1

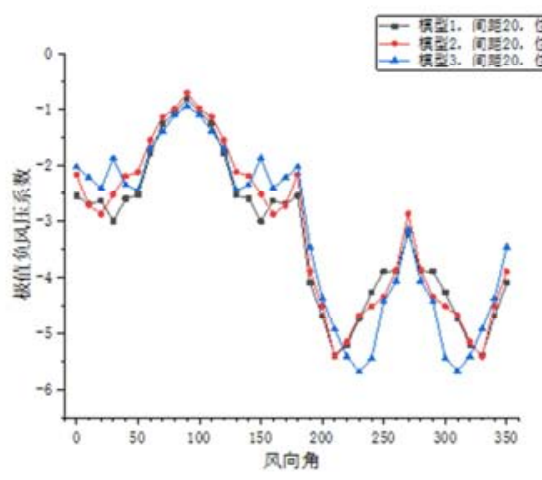

(c) 位置3

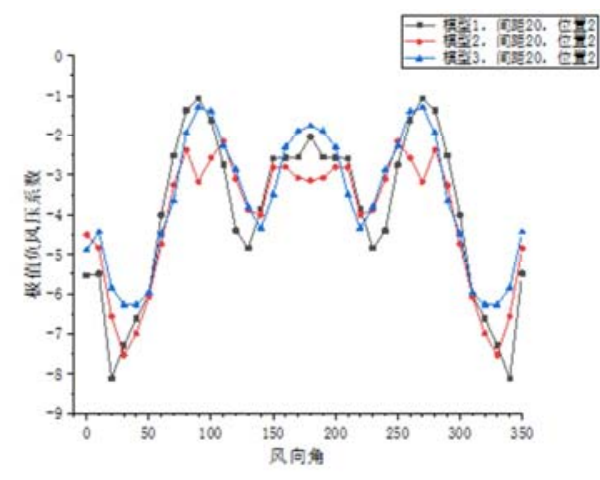

(b) 位置2

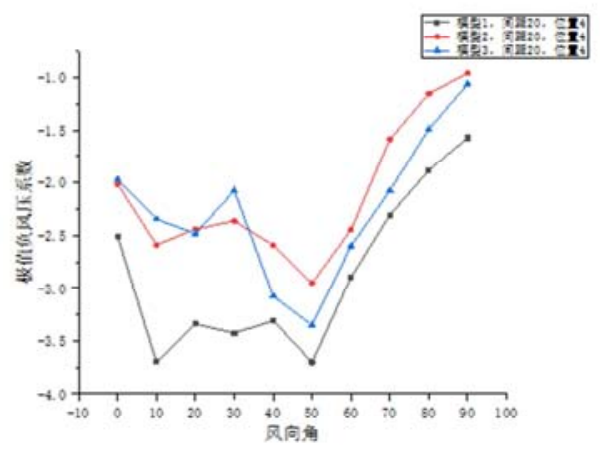

（d）位置4

图11 极值负风压系数随间距和位置变化曲线。 


\section{3. 全工况风压特性分析}

\subsection{1. 极值负风压随风向角变化规律}

图11给出三种长宽比，坡度为 $18.4^{\circ}$ 的建筑物在间距 $20 \mathrm{~m}$ 时, 不同位置下屋面极值负风压系数随风向角变化曲线, 表 2 统计出间距为 $20 \mathrm{~m}$ 时三种长宽比建筑物屋面最不利极值负 风压系数在不同位置的数值、出现的位置及风向角。

从图11可以看出, 不同位置的建筑物屋面极值负风压 系数随风向角变化规律明显不同。位置 1 出现三个最不利 风向角, 分别是 $30^{\circ}, 210^{\circ}, 330^{\circ}$, 位置 2 由于对称性出现 两个最不利风向角, 分别是 $20^{\circ}$ 和 $340^{\circ}$, 位置 3 同样有两个 最不利风向角, 分别是 $230^{\circ}$ 和 $310^{\circ}$, 位置 4 在所有风向角 下的极值负风压系数均小于 -4.0 , 明显小于单体屋面极值 负风压, 因此可不考虑最不利风向角。

最不利极值负风压系数均在位置 1 处最大, 出现的风向 角均为 $30^{\circ}$, 最大值达到- 8.72 , 比单体建筑最不利极值负风 压系数 (-8.19) 要大, 此时对应建筑物长宽比为 $60 \mathrm{~m} \times 12 \mathrm{~m}$ 。 最不利极值负风压系数在位置 4 处最小, 最小值只有 -2.95 , 风向角为 $50^{\circ}$ 附近, 此时对应建筑物长宽比为 $48 \mathrm{~m} \times 12 \mathrm{~m}$; 表 明位置1通常为最不利位置, 位置4为最安全位置;

\subsection{2. 极值负风压随长宽比和间距变化规律}

图12给出间距 $20 \mathrm{~m}$ 时不同位置处的建筑屋面极值负 风压系数随长宽比变化情况。整体上, 全风向下最不利极 值负风压系数从位置1到位置4逐渐减小。随着长宽比的减 小, 位置 $1 、 2$ 的最不利极值负风压系数的数值逐渐减小, 但均大于对应单体建筑的屋面最不利极值负风压系数; 位 置3、4的最不利极值负风压变化不明显, 且均小于对应单 体的屋面最不利极值负风压系数, 表明位置1、2相对位置 $3 、 4$ 而言更为不利。结合表 2 得到, 不同长宽比的建筑在 同一位置时, 屋面最不利极值负风压出现在相同风向角, 在屋面出现的部位也基本一致, 均出现在屋面相同的角部 区域, 表明长宽比的增大并没有改变流场性质, 只是改变 了来流的强度。

图13给出模型一屋面极值负风压系数随间距的变化 曲线。位置1、2处的最不利极值负风压系数随问距增大而 减小，位置3、4处则相反。间距 $20 \mathrm{~m}$ 时，不同位置屋面的 极值负风压系数差距明显，位置 1 和位置 4 相差 $65.6 \%$; 间
距增大为 $50 \mathrm{~m}$ 时, 极值负风压系数变化较小, 尤其位置 1 、 2、3处的最不利极值负风压十分接近, 表明间距越小，屋 面极值负风压对位置的变化更敏感，随着间距的增大，位 置的影响越来越弱。

\subsection{3. 不同分区干扰效应研究}

限于篇幅, 本文以模型 1 为例进行屋面和墙面极值负 风压干扰效应研究。表 2 将模型 1 所有工况下不同分区最大 的极值负风压系数及全风向下最不利干扰因子进行汇总, 图14给出模型1屋面和墙面不同分区最不利极值负风压系 数干扰因子随间距和位置的变化情况。

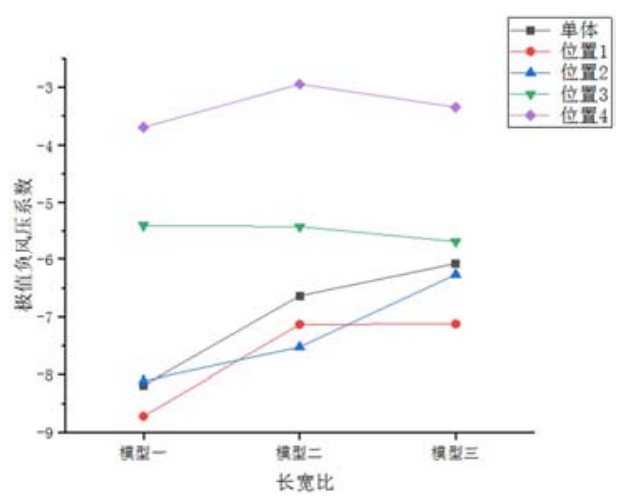

图12 极值负风压随长宽比变化。

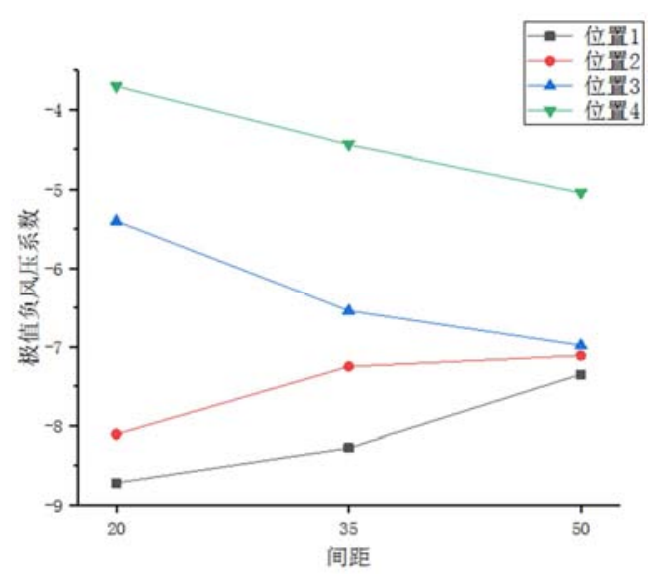

图13 极值负风压随间距变化。

表2 屋面各分区极值负风压系数及干扰因子。

\begin{tabular}{|c|c|c|c|c|c|c|c|c|c|c|}
\hline \multirow{2}{*}{$\begin{array}{c}\text { 间距 } \\
(\mathbf{m})\end{array}$} & \multirow{2}{*}{ 位置 } & \multicolumn{3}{|c|}{ 屋面角部区域 } & \multicolumn{3}{|c|}{ 屋面中部区域 } & \multicolumn{3}{|l|}{ 墙面区域 } \\
\hline & & 极值负风压 & 干扰因子 & 风向角 & 极值负风压 & 干扰因子 & 风向角 & 极值负风压 & 干扰因子 & 风向角 \\
\hline \multirow{4}{*}{20} & 角部位置 & -8.72 & 1.39 & $30^{\circ}$ & -6.69 & 1.19 & $30^{\circ}$ & -5.51 & 1.27 & $350^{\circ}$ \\
\hline & 第一排中间 & -8.11 & 1.31 & $20^{\circ}$ & -5.99 & 1.07 & $20^{\circ}$ & -5.65 & 1.43 & $0^{\circ}$ \\
\hline & 第二排边缘 & -5.40 & 0.87 & $330^{\circ}$ & -5.16 & 0.92 & $320^{\circ}$ & -3.47 & 1.21 & $320^{\circ}$ \\
\hline & 中部位置 & -3.70 & 0.59 & $50^{\circ}$ & -3.45 & 0.61 & $10^{\circ}$ & -3.72 & 1.08 & $30^{\circ}$ \\
\hline \multirow{4}{*}{35} & 角部位置 & -8.28 & 1.32 & $20^{\circ}$ & -6.23 & 1.11 & $20^{\circ}$ & -5.50 & 1.33 & $0^{\circ}$ \\
\hline & 第一排中间 & -7.25 & 1.17 & $30^{\circ}$ & -5.39 & 0.96 & $20^{\circ}$ & -4.91 & 1.33 & $10^{\circ}$ \\
\hline & 第二排边缘 & -6.54 & 1.06 & $330^{\circ}$ & -5.34 & 0.95 & $330^{\circ}$ & -4.83 & 1.69 & $330^{\circ}$ \\
\hline & 中部位置 & -4.44 & 0.59 & $40^{\circ}$ & -3.44 & 0.61 & $50^{\circ}$ & -3.84 & 1.34 & $30^{\circ}$ \\
\hline \multirow{4}{*}{50} & 角部位置 & -7.35 & 1.18 & $30^{\circ}$ & -5.81 & 1.04 & $340^{\circ}$ & -5.74 & 1.40 & $340^{\circ}$ \\
\hline & 第一排中间 & -7.11 & 1.15 & $30^{\circ}$ & -5.27 & 0.94 & $30^{\circ}$ & -4.82 & 1.35 & $0^{\circ}$ \\
\hline & 第二排边缘 & -6.98 & 1.13 & $330^{\circ}$ & -5.67 & 1.01 & $330^{\circ}$ & -4.25 & 1.49 & $340^{\circ}$ \\
\hline & 中部位置 & -5.04 & 0.73 & $50^{\circ}$ & -3.88 & 0.69 & $40^{\circ}$ & -4.18 & 1.46 & $30^{\circ}$ \\
\hline
\end{tabular}




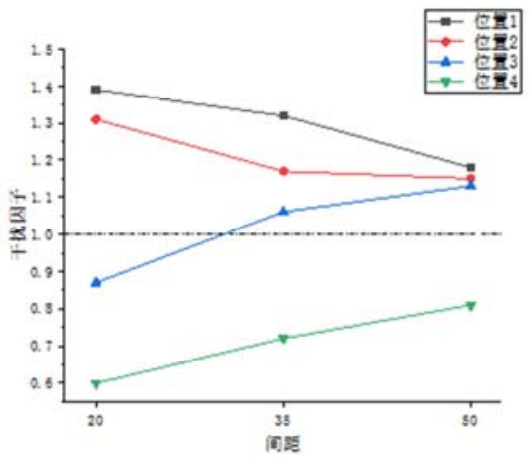

（a）屋面角部区域

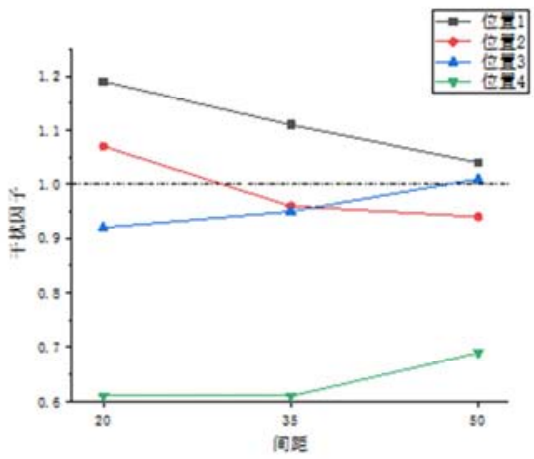

(b) 屋面中间区域

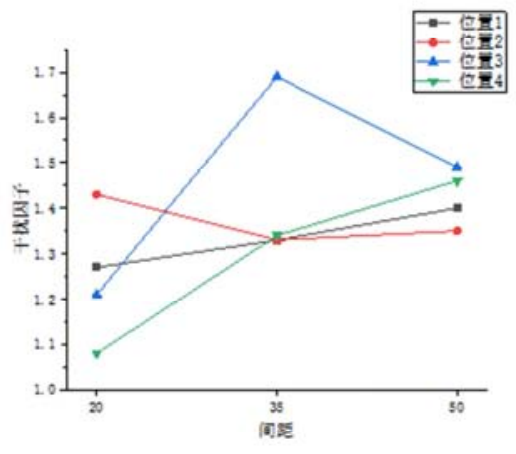

（c）墙面区域

图14 干扰因子随间距变化。

对于屋面角部区域，位置1、2处的干扰因子随间距的 增大而减小, 且始终大于 1.0 , 表明不论间距如何变化, 位置 $1 、 2$ 处建筑屋面角部区域受到的干扰始终为放大效应, 最大干扰因子为 1.39 , 出现在间距 $20 \mathrm{~m}$ 。位置 $3 、 4$ 处的干 扰因子随间距增大而增大, 位置 3 处建筑物当间距为 $20 \mathrm{~m}$ 时, 屋面呈现遮挡效应, 当间距大于 $20 \mathrm{~m}$ 时, 屋面呈现放 大效应; 但对于位置 4 , 干扰因子始终小于 1.0 , 最小为 0.59 , 表明位置4角部区域全程受到遮挡效应, 最大遮挡效应使 屋面角部极值负风压减小 $41 \%$ 。

对于屋面中间区域, 各个位置的干扰因子随间距变化 规律和角部区域一致。间距最小时, 位置1处中间区域最 大干扰因子为 1.19 , 间距 $50 \mathrm{~m}$ 时干扰因子最小为 1.04 , 干 扰呈现放大效应。对于位置 2 , 当间距大于 $20 \mathrm{~m}$ 时, 干扰 因子开始小于 1.0 , 屋面中间区域此时受到遮挡效应。位 置3的干扰因子随间距增大略有增加, 但十分接近 1.0 , 表 明位置3屋面中部区域收到的干扰效应很小。位置4的干扰 因子始终小于 1.0 , 受到最大的遮挡效应为 $39 \%$ 。整体来说, 四个位置的建筑物屋面中间区域较角部位置干扰因子要 小, 更为安全。

对于墙面区域, 不同位置建筑物墙面干扰因子随间距 没有明显的规律, 但是四种位置的最大干扰因子均大于 1.0 , 可能是由于干扰建筑的存在, 各个位置均会在某个 风向角下形成狭道效应, 使墙面的负风压受到不同程度的 放大效应，最大可使墙面的极值负风压放大 $69 \%$ 。

\section{4. 结论}

通过对不同位置, 不同前后间距的的多层小区建筑群 屋面风压和干扰因子进行试验并分析, 得出以下结论:

1. 单体建筑屋面的平均风压和极值风压最不利情况 均出现在屋盖的四个角部和两个端部的屋脊区域。角部位 置为最不利位置, $30^{\circ}, 210^{\circ}, 330^{\circ}$ 为三个最不利风向角, 最不利极值负风压出现在 $30^{\circ}$, 最大为 -8.72 , 原因是 $30^{\circ}$ 屋 盖角部受到气流分离引起的浴旋脱落加上建筑干扰引起 的浴旋两部分共同作用。

2. 由于对称性, 第一排中部位置最不利风向角为 $20^{\circ}$ 和 $340^{\circ}$, 第二排边缘位置最不利风向角为 $230^{\circ}$ 和 $310^{\circ}$, 中 间位置在所有风向角下的极值负风压系数均小于 -4.0 , 明 显小于单体屋面极值负风压, 因此可不考虑最不利风向角。

3. 随着长宽比的减小, 角部和第一排中部位置的最 不利极值负风压系数的数值逐渐减小, 但均大于对应单体 建筑的屋面最不利极值负风压系数; 第二排边缘和中间位 置的最不利极值负风压变化不明显, 且均小于对应单体的 屋面最不利极值负风压系数。角部和第一排中部位置的最 不利极值负风压系数随间距增大而减小, 第二排边缘和中 间位置则相反。

4. 对于屋面角部区域, 角部位置和第一排中部位置 的干扰因子随间距的增大而减小, 且始终大于 1.0 , 最大 
干扰因子为 1.39 , 出现在间距 $20 \mathrm{~m}$ 。第二排边缘和中部位 置的干扰因子随间距增大而增大, 中部位置的干扰因子始 终小于 1.0 。对于屋面中间区域, 各个位置的干扰因子随 间距变化规律和角部区域一致, 但干扰因子整体较角部区 域偏小。墙面区域的干扰因子随间距没有明显的规律，但 干扰因子均大于 1.0 。

\section{致谢}

本研究得到了国家自然科学基金(51678452)的资助, 对此表示感谢。

\section{参考文献}

[1] 乔常贵.中高层住宅区建筑群风干扰效应研究[D], 华侨大学, 2009。

[2] 成俊凯.成纵横排列的群体高层建筑风荷载干扰研究[D], 浙 江大学, 2013。

[3] 全涌, 顾明, 田村幸雄, 黄鹏.周边建筑对低矮建筑平屋面 风荷载的干扰因子 [J]土木工程学报,2010,(2):20-25。

[4] 黄鹏.群体高层建筑风荷载干扰的研究现状及展望.同济大 学学报, 2003, 07, 762-766。

[5] 骆盼育.正交放置平屋盖的风致干扰效应研究[D].北京交通 大学,2014。

[6] 程行, 不同坡度与长宽比双坡屋面低矮建筑群的风荷载干 扰效应研究[D],北京交通大学, 2018。

[7] 中华人民共和国国家标准, 建筑结构荷载规范 GB50009-2012[S].中国建筑出版社.2012。
[8] Tsutsumi J, Katayama T, Nishida M. Wind tunnel tests of wind pressure on regularly alignedbuildings $[\mathrm{J}]$. Journal of Wind Engineering and Industrial Aerodynamics, 1992, 43 (1): 1799-1810.

[9] Yong C K, Yoshida A, Tamura Y. Characteristics of surface wind pressures on low-rise buildinglocated among large group of surrounding buildings [J]. Engineering Structures, 2012, 35: $18-28$.

[10] E. C.English. F. R Frickeb, The interference index and its prediction using a neural network analysis of wind-tunnel data [J]. Journal of Wind Engineering and Industrial Aerodynamics, $1999,83,567-575$.

[11] Yong C K, Tamura Y, Yoon S W. Proximity effect on low-rise building surrounded by similar-sized buildings [J]. Journal of Wind Engineering and Industrial Aerodynamics, 2015, 146: 150-162.

[12] Sun Y, Tamura Y, Quan Y, et al. The interference effect of surrounding roughness on windpressures of rectangular prism [J]. Journal of Bluff Bodies Aerodynamics and Applications, 2008, 6: 1-10.

[13] Chang C H, Meroney R N. The effect of surroundings with different separation distances on surface pressures on low-rise buildings [J]. Journal of Wind Engineering and Industrial Aerodynamics, 2003,91(8): 1039-1050.

[14] Kim Y C, Yoshida A, Tamura Y. Characteristics of surface wind pressures on low-rise building located among large group of surrounding buildings $[\mathrm{J}]$. Engineering Structures, 2012a, 35: 18-28.

[15] Yong Chul Kim, Akihito Yoshida, Yukio Tamura. Influence of Surrounding Buildings on Wind Loads Acting on Low-Rise Building. 2013, 139 (2): 275-283. 